\title{
ON LOGARITHMIC COEFFICIENTS OF CERTAIN STARLIKE FUNCTIONS RELATED TO THE VERTICAL STRIP THE JOURNAL OF ANALYSIS, DOI: 10.1007/S41478-018-0157-7
}

\author{
RAHIM KARGAR
}

\begin{abstract}
In the present paper two certain subclasses of the starlike functions associated with the vertical strip are considered. The main aim of this paper is to investigate some basic properties of these classes such as, subordination relations, sharp inequalities for sums involving logarithmic coefficients and estimate of logarithmic coefficients for functions belonging to these subclasses.
\end{abstract}

\section{IntRODUCTION}

Throughout this paper $\Delta$ is the open unit disc on the complex plane $\mathbb{C}$. Let $\mathcal{H}$ be the class of all analytic functions in $\Delta$ and $\mathcal{A}$ be a subclass of $\mathcal{H}$ with the normalization $f(0)=f^{\prime}(0)-1=0$. The subclass of $\mathcal{A}$ consisting of all univalent functions $f$ in $\Delta$ is denoted by $\mathcal{S}$. Let $\mathcal{S}^{*}$ and $\mathcal{K}$ denote the subclasses of $\mathcal{S}$ consisting of the normalized starlike and convex functions in $\Delta$, respectively. Also, we say that a function $f \in \mathcal{A}$ is close-to-convex, if there is a convex function $g$ such that

$$
\operatorname{Re}\left\{\frac{f^{\prime}(z)}{g^{\prime}(z)}\right\}>0 \quad(z \in \Delta) .
$$

Let $\mathcal{U}(\lambda)$ denote the set of all $f \in \mathcal{A}$ in $\Delta$ satisfying the condition

$$
\left|\left(\frac{z}{f(z)}\right)^{2} f^{\prime}(z)-1\right|<\lambda \quad(z \in \Delta),
$$

where $0<\lambda \leq 1$. For more details and interesting properties of the family $\mathcal{U}(\lambda)$, the reader may refer to [18. Also, let $\mathcal{G}(a)$ denote the class of locally univalent normalized analytic functions $f$ in $\Delta$ satisfying the condition

$$
\operatorname{Re}\left\{1+\frac{z f^{\prime \prime}(z)}{f^{\prime}(z)}\right\}<1+\frac{a}{2} \quad(a>0, z \in \Delta) .
$$

The class $\mathcal{G}(a)$ has been studied extensively by Kargar et al. [11, Maharana et al. [16, Obradović et al. [17, and Ponnusamy and Sahoo 20].

It is well-known that the logarithmic coefficients have had great impact in the development of the theory of univalent functions. For example, de Branges by use of this concept, was able to prove the famous Bieberbach's conjecture [1]. The logarithmic coefficients $\gamma_{n}:=\gamma_{n}(f)$ of $f \in \mathcal{A}$ are defined by

$$
\log \left\{\frac{f(z)}{z}\right\}=\sum_{n=1}^{\infty} 2 \gamma_{n} z^{n} \quad(z \in \Delta) .
$$

As an example consider the rotation of Koebe function

$$
k_{\varepsilon}(z)=\frac{z}{(1-\varepsilon z)^{2}} \quad(|\varepsilon|=1) .
$$

2010 Mathematics Subject Classification. 30C50; 30C45.

Key words and phrases. Univalent; Starlike; Vertical strip; Logarithmic coefficients; Subordination; Hadamard product. 
Then a simple calculation gives that

$$
\gamma_{n}\left(k_{\varepsilon}\right)=\frac{\varepsilon^{n}}{n} \quad(n \geq 1)
$$

The inequality $\left|\gamma_{n}(f)\right| \leq 1 / n$ holds for each starlike function $f \in \mathcal{S}$ and the equality is attained for the rotation of Koebe function, but it is false for the full class $\mathcal{S}$, even in order of magnitude. Let $f \in \mathcal{S}$ and $f(z)=z+\sum_{n=2}^{\infty} a_{n} z^{n}$. Then by (1.1), it follows that

$$
\gamma_{1}=\frac{a_{2}}{2} \quad \text { and } \quad \gamma_{2}=\frac{1}{2}\left(a_{3}-\frac{a_{2}^{2}}{2}\right)
$$

and thus the following sharp estimates hold

$$
\left|\gamma_{1}\right| \leq 1 \quad \text { and } \quad\left|\gamma_{2}\right| \leq \frac{1}{2}\left(1+2 e^{-2}\right) \approx 0.635
$$

However, the sharp estimate of $\left|\gamma_{n}\right|$ when $n \geq 3$ and $f \in \mathcal{S}$ it is still open. For more explanation of this issue, it is necessary to point out that there is a bounded and univalent function with logarithmic coefficients $\gamma_{n}$ such that $\gamma_{n} \neq O\left(n^{-0.83}\right)$ [5. p. 242]. Also, there exists a close-to-convex function $f$ such that $\left|\gamma_{n}(f)\right|>1 / n$, [7. In completing this entry Ye showed that the logarithmic coefficients $\gamma_{n}$ of each close-to-convex function $f$ in $\mathcal{S}$ satisfy $\left|\gamma_{n}(f)\right| \leq(A \log n) / n$, where $A$ is an absolute constant, see 30.

Sharp inequalities are known for sums involving logarithmic coefficients. For instance, the logarithmic coefficients $\gamma_{n}$ of every function $f \in \mathcal{S}$ satisfy the sharp inequality

$$
\sum_{n=1}^{\infty}\left|\gamma_{n}\right|^{2} \leq \frac{\pi^{2}}{6}
$$

and the equality is attained for the Koebe function (see [6, Theorem 4]). Also, for each $f \in \mathcal{S}$ the sharp inequality

$$
\sum_{n=1}^{\infty}\left(\frac{n}{n+1}\right)^{2}\left|\gamma_{n}\right|^{2} \leq 4 \sum_{n=1}^{\infty}\left(\frac{n}{n+1}\right)^{2} \frac{1}{n^{2}}=\frac{2 \pi^{2}-12}{3}
$$

holds (see 24]). Recently, Obradović et al. [19] proved that the logarithmic coefficients $\gamma_{n}$ of any $f \in \mathcal{U}(\lambda)$ satisfy the sharp inequality

$$
\sum_{n=1}^{\infty}\left|\gamma_{n}\right|^{2} \leq \frac{1}{4}\left(\frac{\pi^{2}}{6}+2 L i_{2}(\lambda)+L i_{2}\left(\lambda^{2}\right)\right),
$$

where $L i_{2}$ denotes the dilogarithm function and that the logarithmic coefficients $\gamma_{n}$ of $f \in \mathcal{U}(1)$ satisfy the inequality (1.2). Also, they proved that (see [19, Theorem 2]) the logarithmic coefficients $\gamma_{n}$ of $f \in \mathcal{G}(a)$ satisfy the inequalities

$$
\begin{gathered}
\sum_{n=1}^{\infty} n^{2}\left|\gamma_{n}\right|^{2} \leq \frac{a}{4(a+2)} \quad(0<a \leq 1), \\
\sum_{n=1}^{\infty}\left|\gamma_{n}\right|^{2} \leq \frac{a^{2}}{4} L i_{2}\left((1+a)^{-2}\right) \quad(0<a \leq 1)
\end{gathered}
$$

and

$$
\left|\gamma_{n}\right| \leq \frac{a}{2(a+1) n} \quad(0<a \leq 1, n=1,2, \ldots)
$$

It's worth mentioning, that the above inequality (1.3) is not sharp. Very recently the sharp estimates for the initial logarithmic coefficients $\gamma_{n}$ of $f \in \mathcal{G}(a)$ where $0<a \leq 1$ and $n=1,2,3$ were obtained by Ponnusamy et al. (see [22, Theorem 
2.10]). They, also studied the logarithmic inverse coefficients, denoted by $\Gamma_{n}(F)$, of $f \in \mathcal{G}(a)$, where $F$ is the inverse function of $f \in \mathcal{G}(a)$. For more details see [21].

In the sequel, we recall two certain subclasses of the starlike functions. Let $\mathcal{S}(\alpha, \beta)$ denote the class of all functions $f \in \mathcal{A}$ which satisfy the following twosided inequality

$$
\alpha<\operatorname{Re}\left\{\frac{z f^{\prime}(z)}{f(z)}\right\}<\beta \quad(\alpha<1, \beta>1) .
$$

The class $\mathcal{S}(\alpha, \beta)$ was introduced in [14] and studied in [15] and 29. Also, we expanded the class $\mathcal{S}(\alpha, \beta)$ in [10]. By definition of subordination, $f \in \mathcal{S}(\alpha, \beta)$ if, and only if

$$
\frac{z f^{\prime}(z)}{f(z)} \prec P_{\alpha, \beta}(z) \quad(z \in \Delta)
$$

where

$$
P_{\alpha, \beta}(z):=1+\frac{\beta-\alpha}{\pi} i \log \left(\frac{1-e^{2 \pi i \frac{1-\alpha}{\beta-\alpha}} z}{1-z}\right) .
$$

The function $P_{\alpha, \beta}(z)$ is convex univalent in $\Delta$ and has the form

$$
P_{\alpha, \beta}(z)=1+\sum_{n=1}^{\infty} B_{n} z^{n}
$$

where

$$
B_{n}=\frac{\beta-\alpha}{n \pi} i\left(1-e^{2 n \pi i \frac{1-\alpha}{\beta-\alpha}}\right) \quad(n=1,2, \ldots)
$$

and maps $\Delta$ onto a convex domain

$$
\Omega_{\alpha, \beta}:=\{w \in \mathbb{C}: \alpha<\operatorname{Re} w<\beta\}
$$

conformally. Recently, the function $P_{\alpha, \beta}(z)$ has been studied by many works, see for example 8, 10, 14, 15, 28, 29.

Also, we say that a function $f \in \mathcal{A}$ belongs to the class $\mathcal{M}(\delta)$, if $f$ satisfies

$$
1+\frac{\delta-\pi}{2 \sin \delta}<\operatorname{Re}\left\{\frac{z f^{\prime}(z)}{f(z)}\right\}<1+\frac{\delta}{2 \sin \delta} \quad(z \in \Delta),
$$

where $\pi / 2 \leq \delta<\pi$. The class $\mathcal{M}(\delta)$ was introduced by Kargar et al. 9]. Moreover, by definition of subordination, $f \in \mathcal{M}(\delta)$ if, and only if

$$
\left(\frac{z f^{\prime}(z)}{f(z)}-1\right) \prec \mathcal{B}_{\delta}(z) \quad(z \in \Delta)
$$

where

$$
\mathcal{B}_{\delta}(z):=\frac{1}{2 i \sin \delta} \log \left(\frac{1+z e^{i \delta}}{1+z e^{-i \delta}}\right) \quad(z \in \Delta) .
$$

The function $\mathcal{B}_{\delta}(z)$ due to Dorff [2] and studied in [3], 4], 12] and [13]. The function $\mathcal{B}_{\delta}(z)$ is convex univalent in $\Delta$ and has the form

$$
\mathcal{B}_{\delta}(z)=\sum_{n=1}^{\infty} A_{n} z^{n} \quad(z \in \Delta)
$$

where

$$
A_{n}=\frac{(-1)^{(n-1)} \sin n \delta}{n \sin \delta} \quad(n=1,2, \ldots) .
$$

The following lemma due to Ruscheweyh and Stankiewicz, will be useful in this paper. 
Lemma 1.1. (see [27]) Let $\phi, \varphi \in \mathcal{H}$ be any convex univalent functions in $\Delta$. If $f(z) \prec \phi(z)$ and $g(z) \prec \varphi(z)$, then

$$
f(z) * g(z) \prec \phi(z) * \varphi(z) \quad(z \in \Delta)
$$

where "*" denotes the Hadamard product.

In this paper, some subordination relations among the classes $\mathcal{S}(\alpha, \beta)$ and $\mathcal{M}(\delta)$ are presented. These relations are then used to obtain sharp estimates for sums involving their logarithmic coefficients. Also, the estimate of logarithmic coefficients for functions belonging to these subclasses are determined.

\section{Main Results}

One of the aims of this paper is the following theorem which will be useful in order to estimate of sums involving logarithmic coefficients of functions in the class $\mathcal{S}(\alpha, \beta)$.

Theorem 2.1. Let $f(z) \in \mathcal{A}, \alpha<1$ and $\beta>1$. Also let $P_{\alpha, \beta}(z)$ be defined by (1.5). If $f(z) \in \mathcal{S}(\alpha, \beta)$, then

$$
\log \left\{\frac{f(z)}{z}\right\} \prec \widehat{P}_{\alpha, \beta}(z),
$$

where

$$
\widehat{P}_{\alpha, \beta}(z):=\int_{0}^{z} \frac{P_{\alpha, \beta}(t)-1}{t} \mathrm{~d} t,
$$

and $\widehat{P}_{\alpha, \beta}$ is convex univalent.

Proof. Let $f(z) \in \mathcal{A}$. If we define $p(z):=f(z) / z$, then $p(z)$ is analytic in $\Delta$ and $p(0)=1$. Also, since $f(z) \in \mathcal{S}(\alpha, \beta)$, therefore by (1.4), we have

$$
\frac{z p^{\prime}(z)}{p(z)}=\frac{z f^{\prime}(z)}{f(z)}-1 \prec P_{\alpha, \beta}(z)-1 \quad(z \in \Delta),
$$

where $P_{\alpha, \beta}$ is of the form (1.5). On the other hand, it is well-known that (see [25]) the function

is convex univalent in $\Delta$ and

$$
\widehat{h}(z)=\sum_{n=1}^{\infty} \frac{z^{n}}{n}
$$

$$
\psi(z) * \widehat{h}(z)=\int_{0}^{z} \frac{\psi(t)}{t} \mathrm{~d} t \quad(\psi \in \mathcal{H}) .
$$

Now by Lemma 1.1 and from (2.3) we get

$$
\frac{z p^{\prime}(z)}{p(z)} * \widehat{h}(z) \prec\left(P_{\alpha, \beta}(z)-1\right) * \widehat{h}(z) \quad(z \in \Delta) .
$$

Moreover by (2.4), we can obtain (2.1). On the other hand, since $P_{\alpha, \beta}(z)$ and $\widehat{h}(z)$ are convex univalent functions, by the Pòlya-Schoenberg conjecture (this conjecture states that the class of convex univalent functions is preserved under the convolution) that is proved by Ruscheweyh and Sheil-Small (see [26]), the function $\widehat{P}_{\alpha, \beta}(z)$ is convex univalent, too.

Because $\widehat{P}_{\alpha, \beta}(z)$ is convex univalent, thus we get.

Corollary 2.1. Let $f(z) \in \mathcal{S}(\alpha, \beta)$. Then

$$
\frac{f(z)}{z} \prec \exp \widehat{P}_{\alpha, \beta}(z) \quad(z \in \Delta),
$$

where $\widehat{P}_{\alpha, \beta}(z)$ is given by (2.2). 
Theorem 2.2. For $\alpha<1$ and $\beta>1$, the logarithmic coefficients of $f \in \mathcal{S}(\alpha, \beta)$ satisfy the following inequality

$$
\sum_{n=1}^{\infty}\left|\gamma_{n}\right|^{2} \leq \frac{(\beta-\alpha)^{2}}{4 \pi^{2}}\left(\frac{\pi^{4}}{45}-L i_{4}\left(e^{-2 \pi i \frac{1-\alpha}{\beta-\alpha}}\right)-L i_{4}\left(e^{2 \pi i \frac{1-\alpha}{\beta-\alpha}}\right)\right)
$$

where $\mathrm{Li}_{4}$ is defined as following

$$
L i_{4}(z)=\sum_{n=1}^{\infty} \frac{z^{n}}{n^{4}}=-\frac{1}{2} \int_{0}^{1} \frac{\log ^{2}(1 / t) \log (1-t z)}{t} \mathrm{~d} t .
$$

The result is sharp.

Proof. Let $f \in \mathcal{S}(\alpha, \beta)$. Then by Theorem 2.1, we have

$$
\log \left\{\frac{f(z)}{z}\right\} \prec \widehat{P}_{\alpha, \beta}(z),
$$

where $\widehat{P}_{\alpha, \beta}(z)$ is defined in (2.2). By using (1.6) and (1.7), one can rewrite $\widehat{P}_{\alpha, \beta}(z)$ as the following

$$
\widehat{P}_{\alpha, \beta}(z)=\sum_{n=1}^{\infty} \frac{\beta-\alpha}{\pi n^{2}} i\left(1-e^{2 \pi n i \frac{1-\alpha}{\beta-\alpha}}\right) z^{n} .
$$

With placement of (1.1) and (2.8) into (2.7), we get

$$
\sum_{n=1}^{\infty} 2 \gamma_{n} z^{n} \prec \sum_{n=1}^{\infty} \frac{\beta-\alpha}{\pi n^{2}} i\left(1-e^{2 \pi n i \frac{1-\alpha}{\beta-\alpha}}\right) z^{n} .
$$

Applying Rogosinski's theorem (see [23] or [5, Theorem 6.2]), we obtain

$$
\begin{aligned}
4 \sum_{n=1}^{\infty}\left|\gamma_{n}\right|^{2} & \leq \sum_{n=1}^{\infty} \frac{(\beta-\alpha)^{2}}{\pi^{2} n^{4}}\left|i\left(1-e^{2 \pi n i \frac{1-\alpha}{\beta-\alpha}}\right)\right|^{2} \\
& =\frac{2(\beta-\alpha)^{2}}{\pi^{2}} \sum_{n=1}^{\infty} \frac{1}{n^{4}}\left(1-\cos 2 \pi n \frac{1-\alpha}{\beta-\alpha}\right) \\
& =\frac{2(\beta-\alpha)^{2}}{\pi^{2}}\left(\frac{\pi^{4}}{90}-\frac{1}{2}\left[L i_{4}\left(e^{-2 \pi i \frac{1-\alpha}{\beta-\alpha}}\right)+L i_{4}\left(e^{2 \pi i \frac{1-\alpha}{\beta-\alpha}}\right)\right]\right)
\end{aligned}
$$

and we get the inequality (2.5). The inequality is sharp for the logarithmic coefficients of the function

$$
\mathfrak{F}_{\alpha, \beta}(z)=z \exp \widehat{P}_{\alpha, \beta}(z),
$$

where $\widehat{P}_{\alpha, \beta}(z)$ is given by (2.2). A simple check gives us

$$
\gamma_{n}\left(\mathfrak{F}_{\alpha, \beta}(z)\right)=\frac{\beta-\alpha}{2 \pi n^{2}} i\left(1-e^{2 \pi n i \frac{1-\alpha}{\beta-\alpha}}\right)
$$

and concluding the proof.

Theorem 2.3. Let $f \in \mathcal{A}$ belongs to the class $\mathcal{S}(\alpha, \beta)$ and $\gamma_{n}$ be the logarithmic coefficients of $f$. Then

$$
\left|\gamma_{n}\right| \leq \frac{\beta-\alpha}{n \pi}\left|\sin \frac{\pi(1-\alpha)}{\beta-\alpha}\right| \quad(n \geq 1, \alpha<1<\beta) .
$$

Proof. If $f \in \mathcal{A}$ belongs to the class $\mathcal{S}(\alpha, \beta)$, then by (1.4) we have

$$
z \frac{f^{\prime}(z)}{f(z)}-1=z\left(\log \left\{\frac{f(z)}{z}\right\}\right)^{\prime} \prec P_{\alpha, \beta}(z)-1,
$$


where $P_{\alpha, \beta}$ is defined in (1.5). Moreover, in terms of the logarithmic coefficients $\gamma_{n}$ of $f$ defined by (1.1) and (1.6), is equivalent to

$$
\sum_{n=1}^{\infty} 2 n \gamma_{n} z^{n} \prec \sum_{n=1}^{\infty} B_{n} z^{n}
$$

Now by Rogosinski's theorem (see [23, Theorem X]), we get $2 n\left|\gamma_{n}\right| \leq\left|B_{1}\right|$. Therefore the inequality (2.9) follows. This completes the proof.

It is clear that if $\beta \rightarrow+\infty$, then $\mathcal{S}(\alpha, \beta) \rightarrow \mathcal{S}^{*}(\alpha)$ (the class of starlike functions of order $\alpha$, where $0 \leq \alpha<1$ ). Thus we have the following result (see [19, Remark 1]).

Corollary 2.2. If $f \in \mathcal{S}(\alpha, \beta)$ when $\beta \rightarrow+\infty$, then

$$
\left|\gamma_{n}\right| \leq \frac{\beta-\alpha}{n \pi}\left|\sin \frac{\pi(1-\alpha)}{\beta-\alpha}\right| \leq \frac{\beta-\alpha}{n \pi} \times \frac{\pi(1-\alpha)}{\beta-\alpha}=\frac{1-\alpha}{n} \quad(n \geq 1) .
$$

Indeed, if $f \in \mathcal{S}^{*}(\alpha)(0 \leq \alpha<1)$ and $\gamma_{n}$ is the corresponding logarithmic coeffcients, then we have $\left|\gamma_{n}\right| \leq(1-\alpha) / n$ for $n \geq 1$.

Next, we have the following.

Theorem 2.4. Let $\pi / 2 \leq \delta<\pi$. Also let $\mathcal{B}_{\delta}(z)$ and $A_{n}$ be defined by (1.8) and (1.10), respectively. If $f(z) \in \mathcal{M}(\delta)$, then

$$
\log \left\{\frac{f(z)}{z}\right\} \prec \int_{0}^{z} \frac{\mathcal{B}_{\delta}(t)}{t} \mathrm{~d} t .
$$

Moreover,

$$
\widetilde{\mathcal{B}}_{\delta}(z):=\int_{0}^{z} \frac{\mathcal{B}_{\delta}(t)}{t} \mathrm{~d} t=\sum_{n=1}^{\infty} \frac{A_{n}}{n} z^{n}
$$

is a convex univalent function.

Proof. The proof is similar to the proof of the Theorem 2.1. and thus we omit the details.

Since $\widetilde{\mathcal{B}}_{\delta}(z)$ is a convex univalent function, thus we have.

Corollary 2.3. If $f(z) \in \mathcal{M}(\delta)$, then

$$
\frac{f(z)}{z} \prec \exp \widetilde{\mathcal{B}}_{\delta}(z) \quad(z \in \Delta),
$$

where $\widetilde{\mathcal{B}}_{\delta}(z)$ is of the form (2.10).

Theorem 2.5. Let $f \in \mathcal{A}$ belongs to the class $\mathcal{M}(\delta)$ and $\pi / 2 \leq \delta<\pi$. Then the logarithmic coefficients of $f$ satisfy the inequality

$$
\sum_{n=1}^{\infty}\left|\gamma_{n}\right|^{2} \leq \frac{1}{16 \sin ^{2} \delta}\left[\frac{\pi^{4}}{45}-L i_{4}\left(e^{-2 i \delta}\right)-L i_{4}\left(e^{2 i \delta}\right)\right],
$$

where $\mathrm{Li}_{4}$ is defined in (2.6). The result is sharp.

Proof. Let $f \in \mathcal{M}(\delta)$. Then by Theorem 2.4, we have

$$
\log \left\{\frac{f(z)}{z}\right\} \prec \widetilde{\mathcal{B}}_{\delta}(z) \quad(z \in \Delta) .
$$

By using (1.1) and (1.9), the relation (2.12) implies that

$$
\sum_{n=1}^{\infty} 2 \gamma_{n} z^{n} \prec \sum_{n=1}^{\infty} \frac{A_{n}}{n} z^{n} \quad(z \in \Delta) .
$$


Now by Rogosinski's theorem (see [23] or [5, Theorem 6.2]), we get

$$
\begin{aligned}
4 \sum_{n=1}^{\infty}\left|\gamma_{n}\right|^{2} & \leq \sum_{n=1}^{\infty} \frac{1}{n^{2}}\left|A_{n}\right|^{2} \\
& =\frac{1}{\sin ^{2} \delta} \sum_{n=1}^{\infty} \frac{\sin ^{2} n \delta}{n^{4}} \\
& =\frac{1}{\sin ^{2} \delta} \sum_{n=1}^{\infty}\left(\frac{1}{180}\left[\pi^{4}-45 L i_{4}\left(e^{-2 i \delta}\right)-45 L i_{4}\left(e^{2 i \delta}\right)\right]\right)
\end{aligned}
$$

where $L i_{4}$ is defined by (2.6). Therefore the desired inequality (2.11) follows. For the sharpness of (2.11), consider

$$
F_{\delta}(z)=z \exp \widetilde{\mathcal{B}}_{\delta}(z)
$$

where $\widetilde{\mathcal{B}}_{\delta}(z)$ is defined by (2.10). It is easy to see that $F_{\delta}(z) \in \mathcal{M}(\delta)$ and $\gamma_{n}\left(F_{\delta}\right)=$ $A_{n} / 2 n$, where $A_{n}$ is given by (1.10). Therefore, we have the equality in (2.11). This is the end of proof.

Theorem 2.6. Let $\pi / 2 \leq \delta<\pi$. If $f \in \mathcal{A}$ belongs to the class $\mathcal{M}(\delta)$, then the logarithmic coefficients of $f$ satisfy

$$
\left|\gamma_{n}\right| \leq \frac{1}{2 n} \quad(n \geq 1)
$$

Proof. The proof is similar to the proof of the Theorem 2.3, and thus the details are omitted

Acknowledgements This work is supported by Young Researchers and Elite Club, Ardabil branch. The author would like to thank the anonymous referee(s) for their careful readings, valuable suggestions and comments, which helped to improve the presentation of the paper.

\section{Compliance with ethical standards}

Research involving human participants and/or animals This research does not contain any studies with human participants and/or animals performed by the author.

Conflict of interest The author declares there is no conflict of interest related to this article.

\section{REFERENCES}

[1] De Branges, L.: A proof of the Bieberbach conjecture, Acta Math. 154, 137-152 (1985)

[2] Dorff, M.: Convolutions of planar harmonic convex mappings, Complex Variables, Theory and Appl. 45, 263-271 (2001)

[3] Dorff, M.: Harmonic mappings onto asymmetric vertical strips, in Computational Methods and Function Theory (1997), (N. Papamichael, St. Ruscheweyh and E. B. Saff, eds.), 171-175, World Sci. Publishing, River Edge, NJ, (1999)

[4] Dorff, M., Nowak M., Wołoszkiewicz, M.: Convolutions of harmonic convex mappings, Complex Var. Elliptic Equ. 57, 489-503 (2012)

[5] Duren, P.L.: Univalent Functions, Springer-Verlag, (1983)

[6] Duren, P.L., Leung, Y.J.: Logarithmic coefficients of univalent functions, J. Anal. Math. 36, 36-43 (1979)

[7] Girela, D.: Logarithmic coefficients of univalent functions, Ann. Acad. Sci. Fenn-M. 25, 337$350(2000)$

[8] Kargar, R., Ebadian, A., Sokół, J.: On subordination of some analytic functions, Sib. Math. J. 57, 599-605 (2016)

[9] Kargar, R., Ebadian, A., Sokół, J.: Radius problems for some subclasses of analytic functions, Complex Anal. Oper. Theory 11, 1639-1649 (2017)

[10] Kargar, R., Ebadian, A., Sokół, J.: Some properties of analytic functions related with bounded positive real part, Int. J. Nonlinear Anal. Appl. 8, 235-244 (2017) 
[11] Kargar, R., Pascu, N.R., Ebadian, A.: Locally univalent approximation of analytic functions, J. Math. Anal. Appl. 453, 1005-1021 (2017)

[12] Kumar, R., Gupta, S., Singh, S., Dorff, M.: On harmonic convolutions involving a vertical strip mappings, Bull. Korean Math. Soc. 52, 105-123 (2015)

[13] Kumar, R., Gupta, S., Singh, S., Dorff, M.: An application of Cohn's rule to convolution of univalent harmonic mappings, Rocki Monut. J. Math. 46, 559-570 (2016)

[14] Kuroki, K., Owa, S.: Notes on new class for certain analytic functions, RIMS Kokyuroku Kyoto Univ. 1772, 21-25 (2011)

[15] Kwon, O.S., Sim, Y.J., Cho, N.E., Srivastava, H.M.: Some radius problems related to a certain subclass of analytic functions, Acta Math. Sin. 30, 1133-1144 (2014)

[16] Maharana, S., Prajapat, J. K., Srivastava, H. M.: The radius of convexity of partial sums of convex functions in one direction, Proc. Natl. Acad. Sci., India, Sect. A Phys. Sci. 87, 215-219 (2017)

[17] Obradović, M., Ponnusamy, S., Wirths, K.-J.: Coefficient characterizations and sections for some univalent functions, Sib. Math. J. 54, 679-696 (2013)

[18] Obradović, M., Ponnusamy, S., Wirths, K.-J.: Geometric studies on the class $\mathcal{U}(\lambda)$, Bull. Malays. Math. Sci. Soc. 39, 1259-1284 (2016)

[19] Obradović, M., Ponnusamy, S., Wirths, K.-J.: Logarithmic coefficients and a coefficient conjecture for univalent functions, Monatsh. Math. 185, 489-501 (2018)

[20] Ponnusamy, S., Sahoo, S.K.: Norm estimates for convolution transforms of certain classes of analytic functions, J. Math. Anal. Appl. 342, 171-180 (2008)

[21] Ponnusamy, S., Sharma, N.L., Wirths, K.-J.: Logarithmic coefficients of the inverse of univalent functions, Results Math. (2018) 73: 160. https://doi.org/10.1007/s00025-018-0921-7

[22] Ponnusamy, S., Sharma, N.L., Wirths, K.-J.: Logarithmic coefficients problems in families related to starlike and convex functions, see https://arxiv.org/abs/1811.01203

[23] Rogosinski, W.: On the coefficients of subordinate functions, Proc. London Math. Soc. 48, 48-82 (1943)

[24] Roth, O.: A sharp inequality for the logarithmic coefficients of univalent functions, Proc. Amer. Math. Soc. 135, 2051-2054

[25] Ruscheweyh, St.: New criteria for univalent functions, Proc. Amer. Math. Soc. 49, 109-115 (1975)

[26] Ruscheweyh, St., Sheil-Small, T.: Hadamard product of schlicht functions and the PòylaSchoenberg conjecture, Comm. Math. Helv. 48, 119-135 (1973)

[27] Ruscheweyh, St., Stankiewicz, J.: Subordination under convex univalent function, Bull. Pol. Acad. Sci. Math. 33, 499-502 (1985)

[28] Sim, Y.J., Kwon, O.S.: Certain subclasses of meromorphically bi-univalent functions, Bull. Malays. Math. Sci. Soc. 40, 841-855 (2017)

[29] Sim, Y.J., Kwon, O.S.: Notes on analytic functions with a bounded positive real part, J. Inequal. Appl. 370, 1-6 (2013)

[30] Ye, Z.: The logarithmic coefficients of close-to-convex functions, Bull. Instit. Math. Acad. Sin. (New Series) 3, 445-452 (2008)

Young Researchers and Elite Club, Ardabil Branch, Islamic Azad University, ArdABIL, IRAN

E-mail address: rkargar1983@gmail.com 